\title{
Conservative Management of Subacute Contained Left Ventricular Rupture after Acute Myocardial Infarction
}

\author{
Bharat Kumar Goud $C^{1} \quad$ Johann Christopher ${ }^{2}$ \\ ${ }^{1}$ Department of Cardiology, Nizam's Institute of Medical Sciences, \\ Hyderabad, Telangana, India \\ ${ }^{2}$ Division of Cardiology, Department of Cardiac Imaging, CARE \\ Hospitals, Hyderabad, Telangana, India
}

\begin{abstract}
Address for correspondence Bharat Kumar Goud C, MD, Department of Cardiology, Nizam's Institute of Medical Sciences, F No. 504, Tejaswi Santhiseela Apartments, Beside Telangana Housing Corporation Office, Himayatnagar, Hyderabad-500029, Telangana, India (e-mail: chukkalamd21@gmail.com).
\end{abstract}

\begin{abstract}
Keywords

- subacute

- left ventricular

- free wall rupture

- conservative management

Left ventricular free wall rupture (LVFWR) is a near-fatal mechanical complication of acute myocardial infarction in which an early diagnosis and emergency surgery should be of utmost priority for successful treatment. LVFWR is generally perceived to be universally fatal. Majority of LVFWR patients developing cardiac tamponade die rapidly, while in minority of cases the development of tamponade may be sufficiently slow to allow for diagnosis and successful intervention. In this article, the authors report a case of a 63-year-old male patient diagnosed with an inferoposterior wall myocardial infarction treated with early reperfusion thrombolytic therapy presenting 3 days later with diagnosis of subacute LVFWR. Patient had a history of relapse of chest pain which was severe and prolonged with 2 to $3 \mathrm{~mm}$ saddle-shaped ST-segment elevation in lateral leads, detected on a routine electrocardiogram, which led to an urgent bedside transthoracic echocardiogram (TTE). TTE showed regional wall motion abnormality in form of akinetic basal inferior-wall, a small echodense pericardial effusion, and a canalicular tract from endocardium to pericardium, through which color-Doppler examination suggested blood crossing the myocardial wall. A cardiac magnetic resonance imaging further reinforced the possibility of contained LVFWR.
\end{abstract}

\section{Introduction}

Acute myocardial infarction (AMI) is associated with dreadful but sometimes fatal mechanical complications. Among these, left ventricular free wall rupture (LVFWR) is an infrequent complication (2-4\%), but it is associated with high mortality from pericardial tamponade. ${ }^{1-5}$ LVFWR is presumed to be responsible for as much as 20 to $30 \%$ of all infarct-related deaths. Last two to three decades have witnessed a lot of change epidemiologically with respect to AMI as well as LVFWR. This has been attributed largely to the widespread use of reperfusion therapy. In spite of this change, the large majority of LVFWR may be underreported due to inconsistent autopsy rates. Considering that increased number of patients undergoing primary percutaneous coronary intervention might have led to decreased incidence of LVFWR, ${ }^{6}$ its incidence ranges from 0.7 to $8 \%$. In spite of falling trends of LVFWR, its frequency of occurrence appears to be 8 to 10 times more common than other mechanical complications of MI. ${ }^{6}$

In 1647, William Harvey clinically reported the first postinfarction LVFWR, ${ }^{7}$ but Fitz Gibbon and Montegut in 1972 performed successfully the first operative correction of LVFWR due to AMI.

A prospective study was performed by Figueras et $\mathrm{al}^{8}$ between January 1986 and April 1999 where they studied the efficacy of the conservative management of patients with AMI and clinically suspected free wall rupture, incorporating an alternative surgical approach when medical treatment failed. This study concluded that conservative management of suspicious LVFWR can be successfully done. Other studies have reinforced this conservative management. ${ }^{9}$ 
Here, we describe the case of a 63-year-old patient diagnosed with AMI managed by early thrombolysis presenting 3 days later with contained rupture suspected echocardiographically, confirmed by cardiac magnetic resonance imaging (MRI) and was managed conservatively with follow-up for 6 months with cardiac MRI imaging suggesting spontaneous resolution of contained rupture.

\section{Case Report}

A 63-year-old male patient, who was diagnosed as hypertensive and was on regular medication for the same since then, suffered a cerebrovascular accident several years before and recovered well with no significant residual focal neurological deficit. There was no history of diabetes mellitus, dyslipidemia, tobacco use, or coronary artery disease. He was not on any antiplatelets.

The patient went to the casualty department of a referral hospital with chief complaints of chest pain in left precordial region, which was diffuse and radiating to left arm associated with vomiting and profuse diaphoresis. At presentation, he had rapid thready pulse with raised jugular venous pulsation. The electrocardiogram (ECG) showed features of AMI involving inferolateral wall and posterior wall (-Fig.1). Cardiac biomarkers of necrosis tested positive; the patient was thrombolyzed with streptokinase. Post thrombolytic therapy, patient had symptomatic relief and was shifted to other hospital.

On day 3 after thrombolysis, patient developed chest pain which was more intense than previous episode, was localized to left precordium, but sharper in intensity than before, and occasionally increased in severity by deep inspiration associated with sudden onset of shortness of breath New York Heart Association functional class 4, so he was taken back to the emergency department. On arrival, the ECG showed ventricular tachycardia and patient was hemodynamically unstable. Cardiac rhythm was immediately reverted to sinus rhythm by cardioversion using 200J DC shock. Postcardioversion ECG showed pathological Q waves, with slight $(\sim 1 \mathrm{~mm})$ ST-segment elevation and inverted T waves in the inferior leads.

The patient was admitted to the cardiac care unit (CCU) with the diagnosis of acute inferior wall MI with recurrent angina. He was put on antithrombotic and anti-ischemic medications (including low molecular weight heparin). On admission, he was hemodynamically stable and cardiovascular examination revealed pericardial rub. Two-dimensional transthoracic echocardiogram (TTE) showed regional wall motion abnormality in inferior and lateral wall with mild LV systolic dysfunction and moderate pericardial effusion localized to lateral wall of LV along with thinned-out lateral wall. Doppler interrogation was suggestive of an abnormal blood leak across the inferolateral LV wall and on the basis of these findings, a LVFWR was suspected. Since the patient was complaining of ongoing chest pain, he was referred for urgent coronary catheterization. Coronary angiography was performed at our institution on day 3 after symptom onset which showed a critical stenosis of the left circumflex coronary artery (LCX) with no distal flow, with no other significant coronary lesions. In view of suspicious rupture of free wall, cardiac MRI was done, which revealed myocardial edema in basal and mid inferolateral wall with nonviable transmural scar in LCX territory and contained myocardial rupture in LCX territory ( - Fig.2). Patient was shifted to the CCU for routine monitoring.

The clinical status of patient remained stable. He did not experience any recurrence of chest pain; heart sounds could be heard clearly. there were no murmurs or bruits; and blood pressure (BP) was in an expected range $(\sim 100 / 80 \mathrm{~mm} \mathrm{Hg})$. Serial cardiac biomarkers of necrosis were relatively stable, but white cell counts and C-reactive protein levels were both slightly elevated. A bedside TTE was performed, showing a global small-to-moderate echodense pericardial effusion, somewhat larger in the posterior and inferior aspects of the

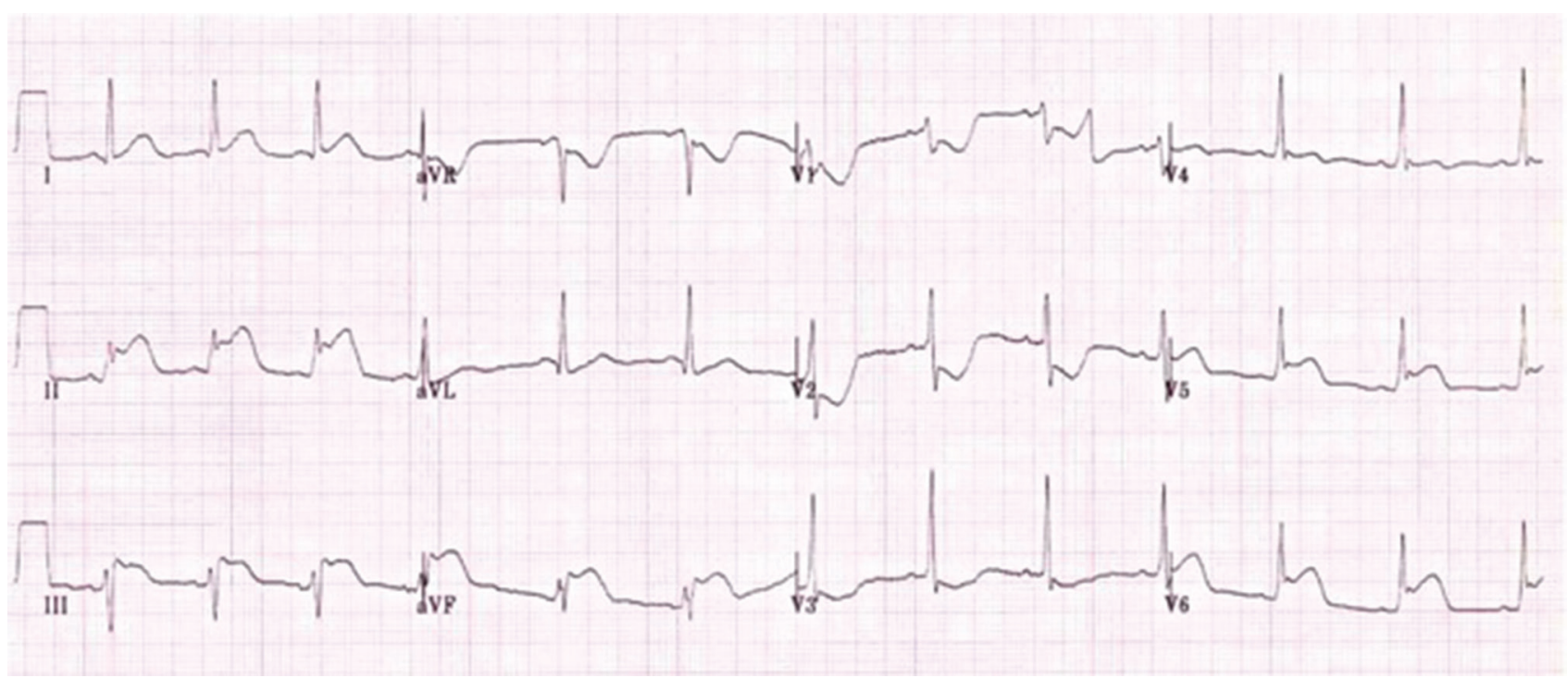

Fig. 1 Electrocardiogram of patient suggestive of acute inferolateral myocardial infarction. 


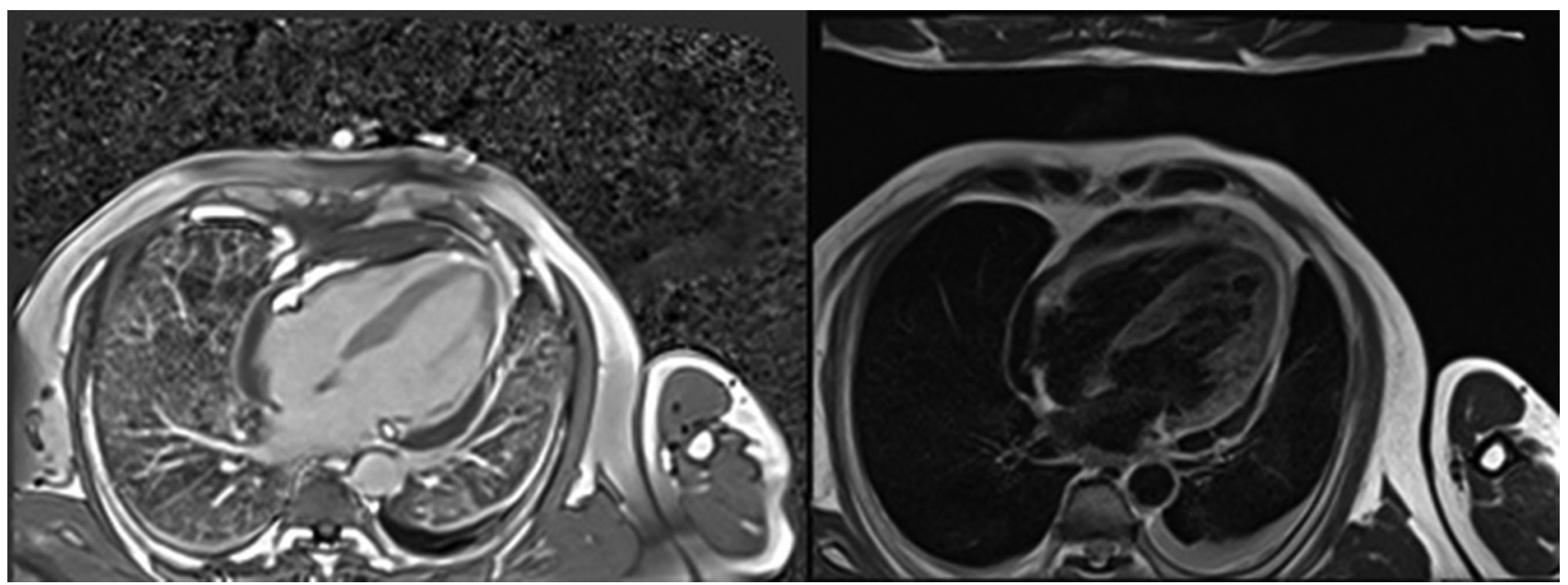

Fig. 2 Cardiac magnetic resonance imaging (A) T2 weighted four chamber image and (B) T1 weighted image of patient showing thinned out nonviable myocardium in inferolateral wall with contained rupture.

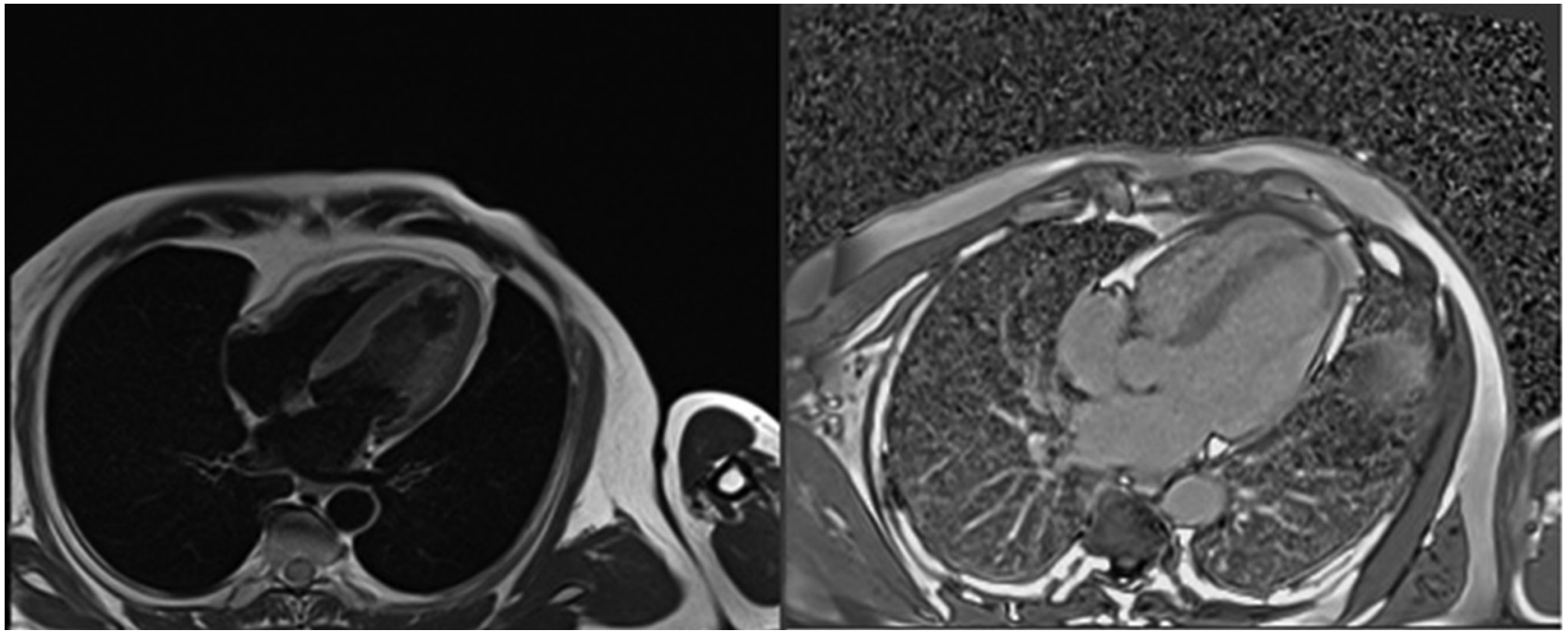

Fig. 3 Cardiac magnetic resonance imaging (A) T1 weighted image and (B) T2 weighted image of same patient four months later showing resolution of contained rupture.

heart, with no signs of tamponade. Patient was not subjected to mechanical revascularization in view of nonviable LCX territory. Patient was discharged with dual antiplatelets, statins, antiarrhythmics, and antihypertensives.

Four months after the event, repeat cardiac MRI showed no evidence of myocardial rupture with nonviable LCX

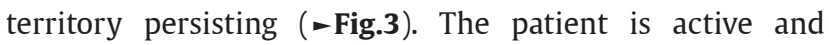
achieved normalcy.

Mechanical complications of AMI apparently tend to become less frequent in day-to-day practice, at least in part due to our growing ability to deliver safe and effective reperfusion therapies (both pharmacological and mechanical) to a wide range of MI patients.

\section{Discussion}

The term subacute rupture was coined by O'Rourke'; however, its definition was not clear. Pollak et $\mathrm{al}^{10}$ defined the "subacute" ruptures as a condition where in the terminal event was preceded by (1) shock with systolic BP of $<80 \mathrm{~mm}$
$\mathrm{Hg}$ and signs of decreased peripheral circulation but survival of $>30$ minutes without cardiopulmonary resuscitation, or (2) intense chest pain of $>30$ minutes duration, which did not respond to nitroglycerin and required alkaloid administration. Ruptures without these prodromal signs were considered as "acute."

Even these definitions were not satisfactory so to make a better distinction between these two terms, Figueras et al ${ }^{11}$ put forward a definition that any cases with moderate-to-severe pericardial effusion and varying severity of hypotension, usually associated with sinus bradycardia or nodal rhythm and raised jugular venous pulsation, be termed as "subacute free wall rupture."

The "subacute free wall rupture" has been recognized frequently and may account for up to $30 \%$ of all cases of in-hospital free wall rupture. ${ }^{1,5}$

The incidence of persistent or recurrent chest pain, hypotension, and ECG changes was $63.5 \%, 94 \%$, and $40 \%$, respectively, in one study. This variation in sensitivity or specificity to diagnose can be improved when all the signs are integrated. 
Patients complaining of prolonged or recurrent post-MI angina if accompanied by spontaneous hypotension should be considered seriously, and evaluated for possible cardiac rupture. ${ }^{5}$ Hypertension on admission also correlated well with cardiac rupture in one study. ${ }^{12}$ In the absence of old MI, patients with these symptoms need an aggressive approach for the prevention of such serious complications. ${ }^{13}$

Hypotension in MI patient is not a highly specific criterion to diagnose LVFWR. In ST elevation MI, hypotension may also result from infarction of right ventricle, severe LV dysfunction, or even dehydration. If the hemorrhage is not hemodynamically significant, BP may be in the normal range. Thus, if hypotension cannot be explained by the known conditions, cardiac tamponade must be taken into account.

The ECG usually suggests a transmural AMI, with a persistently elevated ST segment over the ensuing hours or days. ${ }^{2}$ New ST segment changes may be the chief clinical manifestations. In fact, ECG findings in LV rupture patients may correlate with its type and severity. The acute variety is indicated by electromechanical dissociation (EMD) (with a diagnostic accuracy that reaches $97 \%$ ) and bradycardia, while new ST-elevation in the affected leads or persistent noninversion of T-waves may suggest the less noisy "stuttering" type of rupture.

According to previous studies, cardiac rupture is more common in older aged patients and those with a history of hypertension. Usual age group affected by free wall rupture is between 65 and 70 years, that is, relatively elderly patients, generally older than 55 years. ${ }^{1,45,14}$ There is no apparent sex bias. But in view of the lower incidence of AMI in women, it may be relatively more common in female patients. ${ }^{4,5}$ LVFWR is 8 to 10 times more frequent than rupture of the interventricular septum or papillary muscle. ${ }^{4}$

According to Salem et al, ${ }^{15}$ first 5 days of AMI is the most critical period during which most cases of cardiac rupture $(82 \%)$ occur. Several studies have also shown a similar finding emphasizing the early occurrence of cardiac rupture. ${ }^{3}$

Persistently high BP leading to strain in the infarcted $z^{2} e^{2,14}$ or too early ambulation post-MI may favor this complication. Late rupture generally develops after infarct expansion, while this phenomenon is hardly seen in early rupture. Late rupture can be triggered by persistent vomiting, coughing, or excessive effort when using a bedpan. ${ }^{2}$

Survival advantage with early thrombolytic therapy after AMI has been proved beyond doubt. ${ }^{16}$ Gruppo Italiano per lo Studio della Streptochinasi nell'Infarto (GISSI) study confirmed that thrombolytic treatment increases risk of cardiac rupture. ${ }^{16}$ However, Massel contradicted this finding by showing that there was no strong evidence to suggest that coronary thrombolysis increases overall risk of cardiac rupture. ${ }^{17}$ Although still controversial, there is evidence to suggest that, contrary to data provided by previous reports and post-hoc analysis of pivotal clinical trials, ${ }^{18}$ neither the thrombolysis nor the time delay to thrombolytic treatment actually seems to increase the absolute risk of LVFWR. In the Late Assessment of Thrombolytic Efficacy (LATE) study, Becker et $\mathrm{al}^{19}$ showed that there was no significant difference in the incidence of LVFWR whether a recombinant tissue plasminogen activator-based regimen was given within the 6 to 12 hours period after symptom onset or between 12 and 24 hours. Nakamura et al found that there was statistically significant difference in the incidence of cardiac rupture in the conventionally treated versus in the reperfusion therapy group. ${ }^{20}$ Furthermore, the incidence of cardiac rupture in the unsuccessful reperfusion was higher. They also suggested that early rupture is related to the initial evolution of infarction, whereas late rupture is related to infarct expansion.

Myocardial rupture can be diagnosed by evidence of pericardial effusion which is the most common finding, usually with echodense intrapericardial echoes. The presence as a pericardial effusion in a patient with AMI does not definitively confirm the diagnosis of myocardial rupture, even though its absence excludes the diagnosis. According to one prospective study involving 1,247 MI patients (33 with subacute LV rupture), the presence of cardiac tamponade and pericardial effusion $>5 \mathrm{~mm}$ with high-density intrapericardial echoes or right atrial or right ventricular wall collapse had a high diagnostic sensitivity ( $\geq 70 \%$ ) and specificity ( $\geq 90 \%$ ). When combined with syncope, in the appropriate clinical setting, their diagnostic accuracy may reach $100 \%$.

Until not long ago, there was a thought that surgery was the only definitive treatment option for subacute LVFWR. But recent studies have shown good long-term results following a conservative approach. Che et $\mathrm{al}^{21}$ in 2016 opined that conservative management is an option if patient meets certain criteria. These criteria included patients belonging to older age group and a high surgical risk, who have small infarction with slow bleeding and absence of other mechanical complications. One prospective study reported a $44 \%$ survival rate with conservative management alone among MI patients with confirmed or strongly suspected LVFWR. The study group included patients with AMI who presented with EMD or presented only with hypotension without EMD and were treated conservatively. The main limitation of this study was that there was no comparable control group who underwent surgery and the possibility of other causes of cardiac tamponade could not be definitely ruled out.

\section{Conclusion}

This case illustrates that subacute presentation of LVFWR, which occurs in $30 \%$ of all LVFWR, is not always fatal. In clinical scenario of AMI with persisting angina and ECG changes with or without hypotension, especially in setting of high-risk factors, the diagnosis of LVFWR can be made accurately using bedside echocardiography supplemented by cardiac MRI imaging. The challenge associated with this condition is its unpredictable course, so clinical status of patient cannot be trusted upon and sometimes can even be fatal. Regular monitoring of patients' clinical status with careful conservative management can sometimes yield good results if pericardial effusion is stable. 


\section{References}

1 London RE, London SB. Rupture of the heart. A critical analysis of 47 consecutive autopsy cases. Circulation 1965;31:202-208

2 Friedman HS, Kuhn LA, Katz AM. Clinical and electrocardiographic features of cardiac rupture following acute myocardial infarction. Am J Med 1971;50(6):709-720

3 O'Rourke MF. Subacute heart rupture following myocardial infarction. Clinical features of a correctable condition. Lancet 1973;2(7821):124-126

4 Rasmussen S, Leth A, Kjøller E, Pedersen A. Cardiac rupture in acute myocardial infarction. A review of 72 consecutive cases. Acta Med Scand 1979;205(1-2):11-16

5 Dellborg M, Held P, Swedberg K, Vedin A. Rupture of the myocardium. Occurrence and risk factors. $\mathrm{Br}$ Heart $\mathrm{J}$ 1985;54(1):11-16

6 Yip HK, Wu CJ, Chang HW, et al. Cardiac rupture complicating acute myocardial infarction in the direct percutaneous coronary intervention reperfusion era. Chest 2003;124(2):565-571

7 Willins FA, Dry TJ. A History of the Heart and Circulation. Philadelphia, PA: WB Saunders; 1948

8 Figueras J, Cortadellas J, Soler-Soler J. Left ventricular free wall rupture: clinical presentation and management. Heart. 2000;83(5):499-504

9 Raitt MH, Kraft CD, Gardner CJ, Pearlman AS, Otto CM. Subacute ventricular free wall rupture complicating myocardial infarction. Am Heart J 1993;126(4):946-955

10 Pollak H, Diez W, Spiel R, Enenkel W, Mlczoch J. Early diagnosis of subacute free wall rupture complicating acute myocardial infarction. Eur Heart J 1993;14:640-648

11 Figueras J, Curos A, Cortadellas J, Sans M, Soler-Soler J. Relevance of electrocardiographic findings, heart failure, and infarct site in assessing risk and timing of left ventricular free wall rupture during acute myocardial infarction. Am J Cardiol. 1995;76:543-547
12 Nakano T, Konishi T, Takezawa H. Potential prevention of myocardial rupture resulting from acute myocardial infarction. Clin Cardiol 1985;8(4):199-204

13 Reddy SG, Roberts WC. Frequency of rupture of the left ventricular free wall or ventricular septum among necropsy cases of fatal acute myocardial infarction since introduction of coronary care units. Am J Cardiol 1989;63(13):906-911

14 Naeim F, De la Maza LM, Robbins SL. Cardiac rupture during myocardial infarction. A review of 44 cases. Circulation 1972;45(6):1231-1239

15 Salem BI, Lagos JA, Haikel M, Gowda S. The potential impact of the thrombolytic era on cardiac rupture complicating acute myocardial infarction. Angiology 1994;45:931-936

16 Gruppo Italiano per lo studio della streptochinasi nell' infarto miocardico (GISSI): Effectiveness of intravenous thrombolytic treatment in acute myocardial infarction. Lancet 1986;1:397-402

17 Massel D. Cardiac rupture and time to thrombolytic treatment. J Am Coll Cardiol 1991;17(7):1671-1672

18 Maggioni AP, Maseri A, Fresco C, et al. Age-related increase in mortality among patients with first myocardial infarctions treated with thrombolysis. The Investigators of the Gruppo Italiano per lo Studio della Sopravvivenza nell'Infarto Miocardico (GISSI-2) N Engl J Med 1993;329(20):1442-1448

19 Becker RC, Charlesworth A, Wilcox RG, et al. Cardiac rupture associated with thrombolytic therapy: impact of time to therapy in the Late Assessment of Thrombolytic Efficacy (LATE) study. J Am Coll Cardiol 1995;25:1063-1068

20 Nakamura F, Minamino T, Higashino Y, et al. Cardiac free wall rupture in acute myocardial infarction: ameliorative effect of coronary reperfusion. Clin Cardiol 1992;15(4):244-250

21 Che J, Li G, Chen K, Liu T. Post-MI free wall rupture syndrome. Case report, literature review, and new terminology. Clin Case Rep 2016;4(6):576-583 